\title{
Cost-Sensitive Deep Active Learning for Epileptic Seizure Detection
}

\author{
Xuhui Chen \\ Case Western Reserve University \\ Cleveland, Ohio \\ xxc296@case.edu \\ Tianxi Ji \\ Case Western Reserve University \\ Cleveland, Ohio \\ txj116@case.edu
}

\author{
Jinlong Ji \\ Case Western Reserve University \\ Cleveland, Ohio \\ jxj405@case.edu \\ Pan Li \\ Case Western Reserve University \\ Cleveland, Ohio \\ pxl288@case.edu
}

\begin{abstract}
The analysis of electroencephalogram (EEG) signal plays a crucial role in epileptic seizure detection. Researchers have proposed many machine learning and deep learning based automatic epileptic seizure detection methods. However, these schemes, especially the deep learning based ones, suffer from labeling huge amounts of training data. Moreover, in epileptic seizure detection, physicians pay more attention to abnormal signals than normal signals, and thus the misclassification cost for them should be different. To address these issues, we propose a cost-sensitive deep active learning scheme to detect the epileptic seizure. In particular, we develop a new generic double-deep neural network (double-DNN) to obtain the cost-sensitive utility for the samples selection strategy in the labeling process. We further employ three types of fundamental neural networks, i.e., one-dimensional convolutional neural networks (1D CNNs), recurrent neural networks with long short-term memory (LSTM) units, and recurrent neural networks with gated recurrent units (GRU), in the doubleDNN and evaluate their performances. Experiment results show that the proposed scheme can reduce the amount of labeled samples by up to $33 \%$ and $80 \%$ compared with uncertainty sampling and random sampling, respectively.
\end{abstract}

\section{CCS CONCEPTS}

- Theory of computation $\rightarrow$ Theory and algorithm$\mathrm{s}$ for application domains; Machine learning theory; Active learning;

Permission to make digital or hard copies of all or part of this work for personal or classroom use is granted without fee provided that copies are not made or distributed for profit or commercial advantage and that copies bear this notice and the full citation on the first page. Copyrights for components of this work owned by others than ACM must be honored. Abstracting with credit is permitted. To copy otherwise, or republish, to post on servers or to redistribute to lists, requires prior specific permission and/or a fee. Request permissions from permissions@acm.org.

ACM-BCB'18, August 29-September 1, 2018, Washington, DC, USA

(C) 2018 Association for Computing Machinery.

ACM ISBN 978-1-4503-5794-4/18/08 . .\$15.00

https://doi.org/10.1145/3233547.3233566

\section{KEYWORDS}

Active Learning; Deep Learning; EEG; Epileptic Seizure Detection

\section{ACM Reference Format:}

Xuhui Chen, Jinlong Ji, Tianxi Ji, and Pan Li. 2018. Cost-Sensitive Deep Active Learning for Epileptic Seizure Detection. In $A C M$ BCB'18: 9th ACM International Conference on Bioinformatics, Computational Biology and Health Informatics, August 29September 1, 2018, Washington, DC, USA, Jennifer, Theo D'Hondt, and Wolfgang De Meuter (Eds.). ACM, New York, NY, USA, Article 4, 10 pages. https://doi.org/10.1145/3233547.3233566

\section{INTRODUCTION}

An estimated population of 65 million people worldwide currently live with epilepsy, which is one of the most common neurological disorders. Epileptic seizures may cause convulsions, muscle spasms, brief or prolonged loss of consciousness, strange sensations and emotions, and abnormal behaviors [10]. The analysis of electroencephalogram (EEG), a multichannel recording of the brain's electrical activity, plays a crucial role in detecting the seizure onset. Traditionally, this process is manually conducted by physicians based on their professional knowledge and medical experiences. However, detecting seizure onset is extremely time and effort consuming when the EEG data is large. To relieve this burden, researchers have proposed many machine learning based automatic analyzing methods, such as support vector machine (SVM) [2], artificial neural network (ANN) [12, 33], decision tree (DT) [34]. As deep learning schemes show outstanding performances in computer vision, speech recognition, and natural language processing $[22,27]$, researchers also attempt to utilize deep neural networks (DNNs) to detect the epileptic seizure onset and achieve good performances [38].

However, training a deep learning model usually requires large amounts of annotated data, the collection of which is very challenging and expensive. Particularly, labeling EEG signals not only requires the expertise of an experienced pathologist but also is very time-consuming. On the contrary, unlabeled data is easy to obtain but hard to use in the training process. In this paper, we take advantage of active learning to address this issue. The goal of active learning is to obtain high prediction accuracy by using as few labeled 
training samples as possible. In contrast to supervised learning, active learning interactively requests a portion of the most informative instances to be labeled by an oracle (e.g., a physician or an expert) during the classifier training process, and thus reducing the human labeling cost significantly [36].

The main challenge for active learning is to design a proper metric to evaluate the utility of the samples in the unlabeled data pool. In the literature, most studies on the sampling strategy are cost-insensitive and treat all the misclassifications the same. However, in many applications, especially in medical data analysis, the effects of misclassifications on data samples are different. For instance, in seizure detection, the misclassification of a normal signal is less critical than that of an abnormal signal since the latter misclassification may cause severe medical negligence.

Therefore, we take misclassification cost into consideration and propose a cost-sensitive deep active learning framework for epileptic seizure detection. We introduce a new double-deep neural network (double-DNN) to learn actively, where the primary DNN is to give the seizure prediction results, while the assistant DNN predicts the misclassification cost of each sample in the unlabeled data pool. Our double-DNN is a generic structure, which can be implemented with any type of neural layers. In this paper, we employ one-dimensional convolutional neural network (1-D CNN), recurrent neural network with long short-term memory (LSTM) units, and recurrent neural network with gated recurrent units (GRU) as examples to evaluate the proposed framework and compare their performances. Our main contributions can be briefly summarized as follows:

- We are the first to develop deep learning networks with active learning for epileptic seizure detection using EEG signals.

- We take misclassification cost into consideration and propose a double-DNN system to perform cost-sensitive deep active learning.

- The experiment results validate the efficiency and efficacy of the proposed scheme for epileptic seizure detection.

The rest of this paper is organized as follows. We first give a brief introduction to active learning and machine learning based seizure detection schemes in Section 2, and introduce some preliminary of neural networks for time series analysis in Section 3. Then we describe the proposed double-DNN system in Section 4. To demonstrate the efficacy and efficiency of our system, we present the experiment results with evaluations in Section 5. Finally, we conclude this paper in Section 6 .

\section{RELATED WORKS}

\subsection{Machine Learning and Deep Learning for EEG Analysis}

Many machine learning algorithms have been developed for epilepsy detection by utilizing SVM, ANN, decision tree, etc
$[1,6,29]$. Based on the property of epilepsy, Srinivasan et al. [39] adopt the approximate entropy feature to measure the predictability of EEG signals between current amplitude and the previous amplitude values. ApEn is a statistical parameter that calculates the regularity of signal in time domain. Srinivasan et al. [39] also propose a similar algorithm but both time domain and frequency domain features. The network used in both works is recurrent Elman neural networks. Acharya et al. [1] focuses on more entropy features, such as Approximate Entropy (ApEn), Sample Entropy (SampEn), and two Phase Entropy, and also explore more comprehensive applications by feeding the entropy features to seven different classifiers: Fuzzy Sugeno Classifier, Support Vector Machine, K-Nearest Neighbour, Probabilistic Neural Network, Decision Tree, Gaussian Mixture Model, and Naive Bayes Classifier.

Besides, some works have applied deep learning to EEG signal classification. Zheng et al. [46] employ a deep belief network (DBN) to classify emotions using EEG signals. Extracted differential entropy features from multichannel EEG signals are fed as input to train the deep belief network. Subsequently, the output was integrated with a hidden Markov model (HMM) for the detection of emotional stage switching. An et al. [4] demonstrate a deep learning algorithm for motor imagery EEG classification, where both left and righthand motor imagery data are studied. First, a series of weak classifiers are trained by DBN using each single channel data. Then the AdaBoost algorithm combines the weak classifiers to obtain a strong classifier. In particular, a restricted Boltzman machine (RBM) formes a parallel layer DBN structure. The contrastive divergence (CD) algorithm is also employed to achieve higher accuracy. .

With recent advances in deep learning, researchers have analyzed EEG signals by utilizing many modern neural networks, such as Recurrent Neural Networks (RNN) [9, 16, 21], and Convolutional Neural Networks (CNN) [35, 41]. Particularly, Stober et al. [40] input the EEG signals directly into a CNN to classify individual rhythms. They demonstrate that perceived rhythms can be identified from EEG data during their auditory presentation using CNNs that look only at a short segment of the signals from a single EEG channel.

\subsection{Active Learning}

Active learning has been widely applied in many areas, which aims to label only the most informative data and thus reduces the labeling efforts significantly. Wan et al. [42], Liu et al. [30], and Gal et al [18] apply active learning to image recognition and classification. As shown in their works, using active learning can lead to promising performance with much less training data. Other applications of active learning include functioning optimization [32] and experimental design optimization [6].

With success in diverse fields, active learning also draws attention on EEG analysis. Wu et al. [43] study single-trial 
visual-evoked potential (VEP) classification using EEG signals. This work utilizes active learning techniques for artifact detection and identification in EEG signals. They later extend their work from binary classification to multi-class classification [25]. Their experiment results show that by using active learning, the same classification accuracy can be achieved with $25 \%$ of the labeled data. Wu et al. [44] explore brain-computer interface (BCI) applications and propose an enhanced batch-mode active learning (EBMAL) approach for regression problems, which improves a baseline active learning algorithm by increasing the reliability, representativeness, and diversity of the selected samples to achieve better regression performance. They validate its effectiveness through driver drowsiness detection based on EEG signals. The authors further study the active weighted adaptation regularization (AwAR), which integrates weighted adaptation regularization (wAR) and active learning, to expedite the calibration process [45]. Experiments on single-trial event-related potential classification show that AwAR can significantly increase the classification accuracy, given the same number of labeled samples from the dataset. Jia et al. [23] propose a semi-supervised deep structured framework to recognize the affective state using EEG signals. They first adopt a two-level procedure involving both supervised label information and unsupervised structure information to jointly make a decision on channel selection. Then, they add a generative Restricted Boltzmann Machine (RBM) model for the classification task and use the training objectives of generative learning and unsupervised learning to regularize the discriminative training jointly. Finally, they extend it to the active learning scenario, which solves the costly labeling problem. The experiments have shown both convincing results on critical channel selection and superiority of the method over multiple baselines.

Moreover, there are some works on active learning in seizure detection. Balakrishnan and Syed [7] are the first to apply active learning to detecting seizure onset that using a support vector machine-based active learning algorithm. Experiments show that the approach reduces the amount of labeled data by nearly $95 \%$. They extend the work to compare different approaches for initialization, batch selection and termination in the active learning process [8]. Results show that when using a combined measurement of distance and diversity in their sampling strategy, an active learning-based detector needs much fewer labeled examples for training.

\section{PRELIMINARY}

Deep learning is a remarkably effective machine learning tool for many applications [26]. It attempts to learn a predictive model from inputs to outputs by employing deep neural networks (DNNs). In general, DNNs have multiple layers of nonlinear processing nodes, which enable the networks to learn feature representations layer by layer.

In this paper, we focus on EEG signal analysis, in which the data are always captured over the course of time, constituting a sequence-series. Traditional DNNs lack the ability to model the dynamic temporal behavior inside such data. Then, many researchers propose many DNN variants to capture data's temporal features, like 1D CNNs [20], RNNs [31], LSTMs [19], and GRUs [13]. In what follows, we introduce them briefly, which will be the candidate building blocks in our system design.

\subsection{D CNN}

CNNs are initially designed for computer vision and have achieved success in many other fields [27]. It stacks many layers of convolutional filters and pooling operations on top of each other. Therefore, it is able to extract the representations of the inputs. Particularly, 1D CNNs utilize filters to convolve data along one specific dimension, which enables it to analyze time series data along the temporal dimension.

We denote a time sequence data by

$$
x_{1: n}=x_{1} \oplus x_{2} \oplus \cdots \oplus x_{n},
$$

where $x_{i} \in \mathbb{R}$ is a data point in the sequence, $\oplus$ is the concatenation operator, and $n$ is the length. In general, let $x_{i: i+j}$ refer to a subsequence $x_{i} \oplus x_{i+1} \oplus \cdots \oplus x_{i+j}$.

A 1D convolutional filter $\mathbf{w} \in \mathbb{R}^{h \times 1}$ is applied to a sliding window of length $h$ on the sequence data to generate a new feature $c$. For instance, a feature $c_{i}$ is obtained from a window of data points $x_{i: i+h-1}$ by

$$
c_{i}=f\left(\mathbf{w} \cdot x_{i: i+h-1}+b\right),
$$

where $b \in \mathbb{R}$ is bias and $f(\cdot)$ is a non-linear activation function. Then we apply the filter to each possible window of data points to generate a feature map c:

$$
\mathbf{c}=\left[c_{1}, c_{2}, \cdots, c_{n-h+1}\right],
$$

with $\mathbf{c} \in \mathbb{R}^{n-h+1}$

We then apply 1D max-pooling operation [15] over the feature map and take the maximum value $\hat{c}=\max \{\mathbf{c}\}$ as the feature corresponding to this particular filter, which captures the most important feature in the feature map.

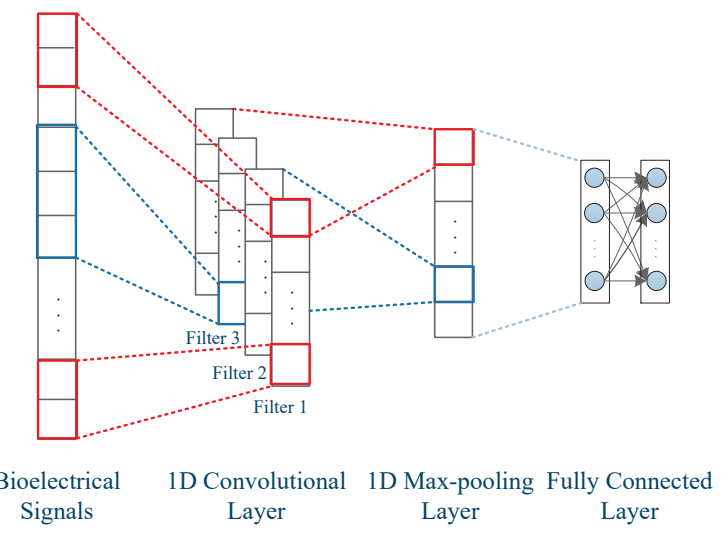

Figure 1: The architecture of $1 \mathrm{D}$ CNNs

As shown in Fig. 1, a $1 \mathrm{D}$ CNN uses multiple filters to obtain multiple features. After that, the networks utilize a 
fully-connected softmax layer to generate the probability distribution over labels.

\subsection{Recurrent Neural Networks}

RNN [17] is proposed to process sequence data by recursively applying a transition function to its internal hidden state vector $\mathbf{h}_{t}$ of the input sequence, as shown in Fig. 2.

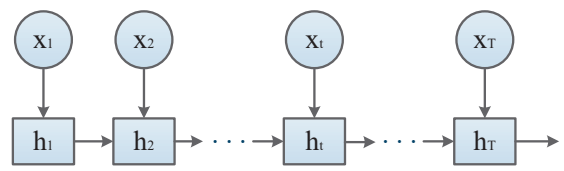

Figure 2: The architecture of RNN

Particularly, the hidden state vector $\mathbf{h}_{t}$ is computed by the current input data $x_{t}$ and the previous hidden state $\mathbf{h}_{t-1}$ :

$$
\mathbf{h}_{t}=\left\{\begin{array}{ll}
0 & t=0 \\
f\left(\mathbf{h}_{t-1}, x_{t}\right) & \text { otherwise }
\end{array},\right.
$$

where $f(\cdot)$ is the an element-wise nonlinear activation function.

Traditionally, a simple strategy for classifying sequence data is to map the data to a hidden state vector using one RNN. The vector is then fed into fully-connected layers with softmax output layer for classification.

RNNs can successfully process sequence data in many applications. However, a standard RNN suffers from gradients vanishing or exploding dealing with long sequences. To address this issue, researchers develop LSTM units and GRU units, which can learn long-term dependencies effectively. In the following, we illustrate the main ideas of these two networks, respectively.

3.2.1 RNN with LSTM Units. We replace the cells in naive RNNs with LSTM units as shown in Fig. 3. The details of the cells are as follows:

$$
\begin{aligned}
\mathbf{i}_{t} & =\sigma\left(\mathbf{W}_{i x} \mathbf{x}_{t}+\mathbf{W}_{i h} \mathbf{h}_{t-1}+\mathbf{W}_{i c} \mathbf{c}_{t-1}+\mathbf{b}_{i}\right) \\
\mathbf{f}_{t} & =\sigma\left(\mathbf{W}_{f x} \mathbf{x}_{t}+\mathbf{W}_{f h} \mathbf{h}_{t-1}+\mathbf{W}_{f c} \mathbf{c}_{t-1}+\mathbf{b}_{f}\right) \\
\mathbf{c}_{t} & =\mathbf{f}_{t} \odot \mathbf{c}_{t-1}+\mathbf{i}_{t} \odot \phi\left(\mathbf{W}_{c x} \mathbf{x}_{t}+\mathbf{W}_{c h} \mathbf{h}_{t-1}+\mathbf{b}_{c}\right) \\
\mathbf{o}_{t} & =\sigma\left(\mathbf{W}_{o x} \mathbf{x}_{t}+\mathbf{W}_{o h} \mathbf{h}_{t-1}+\mathbf{W}_{o c} \mathbf{c}_{t}+\mathbf{b}_{o}\right) \\
\mathbf{h}_{t} & =\mathbf{o}_{t} \odot \phi\left(\mathbf{c}_{t}\right)
\end{aligned}
$$

Specifically, at time instance $t$, each LSTM unit is constructed by an input gate $\mathbf{i}_{t}$, a forget gate $\mathbf{f}_{t}$, an output gate $\mathbf{o}_{t}$, and a memory cell $\mathbf{c}_{t}$. These gates and memory cell have the same dimension in $\mathbb{R}^{d}$ as the hidden vector $\mathbf{h}_{t}$. The cel$\mathbf{l}$ is activated by current inputs $\mathbf{x}_{t}\left(\mathbf{x}_{t} \in \mathbb{R}^{e}\right)$ and previous hidden state $\mathbf{h}_{t-1} . \mathbf{W}_{i x}, \mathbf{W}_{i h}, \mathbf{W}_{i c}, \mathbf{W}_{f x}, \mathbf{W}_{f h}, \mathbf{W}_{f c}, \mathbf{W}_{o x}$, $\mathbf{W}_{o h}, \mathbf{W}_{o c} \in \mathbb{R}^{d \times e}$ are weighted matrices, and $\mathbf{b}_{i}, \mathbf{b}_{f}, \mathbf{b}_{c}, \mathbf{b}_{o}$ $\in \mathbb{R}^{d}$ are biases of LSTM, which are all learned during training process. $\sigma$ is the sigmoid function and $\odot$ stands for element-wise multiplication.
The update of each LSTM unit can be briefly summarized as follows:

$$
\mathbf{h}_{t}=\operatorname{LSTM}\left(\mathbf{h}_{t-1}, \mathbf{x}_{t}, \theta\right),
$$

where function $\operatorname{LSTM}(\cdot)$ represents Eq. (5-9), and $\theta$ denotes all the parameters in the LSTM unit.

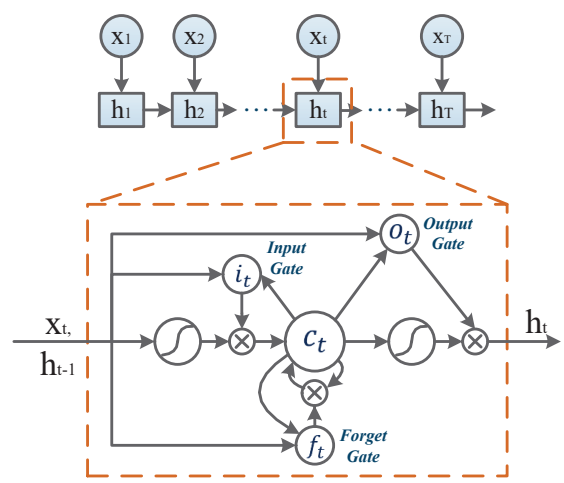

Figure 3: The architecture of RNN with LSTM units.

3.2.2 RNNs with GRU Units. We replace the cells in naive RNNs with GRU units [14] as shown in Fig. 4. The details of the cells are as follows:

$$
\begin{aligned}
\mathbf{z}_{t} & =\sigma\left(\mathbf{W}_{h z} \mathbf{h}_{t-1}+\mathbf{W}_{x z} \mathbf{x}_{t}\right) \\
\mathbf{r}_{t} & =\sigma\left(\mathbf{W}_{h r} \mathbf{h}_{t-1}+\mathbf{W}_{x r} \mathbf{x}_{t}\right) \\
\mathbf{c}_{t} & =\tanh \left(\mathbf{W}_{c}\left(\mathbf{h}_{t-1} \odot \mathbf{r}\right)+\mathbf{U}_{c} \mathbf{x}_{t}\right) \\
\mathbf{h}_{t} & =(\mathbf{z} \odot \mathbf{c})+\left(\left(1-\mathbf{z}_{t}\right) \odot \mathbf{h}_{t-1}\right)
\end{aligned}
$$

Particularly, at time instance $t$, each GRU unit is constructed by an update gate $\mathbf{x}_{t}$ and a reset gate $\mathbf{r}_{t}$. These gates have the same dimension in $\mathbb{R}^{d}$ as the candidate hidden vector $\mathbf{c}_{t}$ and hidden vector $\mathbf{h}_{t}$. The cell is activated by current input $\mathbf{x}_{t}\left(\mathbf{x}_{t}=x_{i_{t}: i_{t+e-1}}\right)$ and the previous hidden state $\mathbf{h}_{t-1} . \mathbf{W}_{h z}, \mathbf{W}_{x z}, \mathbf{W}_{h r}, \mathbf{W}_{x r}, \mathbf{W}_{c}$ and $\mathbf{U}_{c} \in \mathbb{R}^{d \times e}$ are weighted matrices, which are learned during training process. $\sigma(\cdot)$ and $\tanh (\cdot)$ are the activation function and $\odot$ stands for element-wise multiplication.

The update of each GRU unit can be briefly summarized as:

$$
\mathbf{h}_{t}=\operatorname{GRU}\left(\mathbf{h}_{t-1}, \mathbf{x}_{t}, \mathbf{w}_{t}\right)
$$

where function GRU (.) denotes Eq. (11-14), and $\mathbf{w}_{t}$ represents all the parameters in the GRU unit.

In this paper, for both RNN with LSTM units and that with GRU unites, we input an EEG segment $\mathbf{x}=\left\{x_{1}, x_{2}, \ldots, x_{T}\right\}$ to the RNN layers to output a representation vector $\mathbf{h}_{T}$. Finally, $\mathbf{h}_{T}$ is input into a fully connected layer to generate a probability distribution over all classes, i.e.,

$$
\hat{\mathbf{y}}=\operatorname{sigmoid}\left(\mathbf{W h}_{T}+\mathbf{b}\right)
$$

where $\hat{\mathbf{y}}$ is prediction probabilities, $\mathbf{W}$ is the learned weights, and $\mathbf{b}$ is the bias. For a given classic classification task, the 


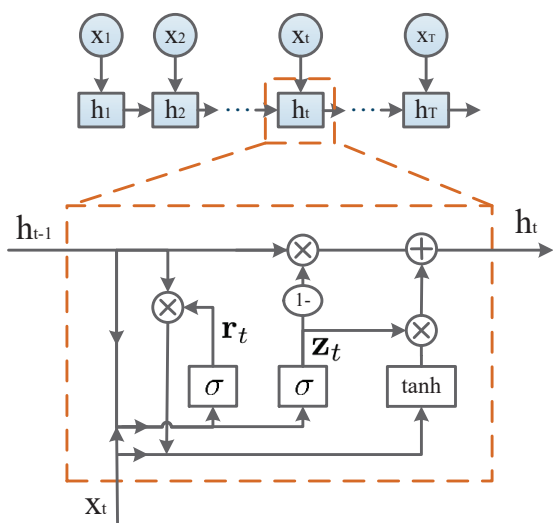

Figure 4: The architecture of RNN with GRU units.

loss function is defined as the cross-entropy between predicted and ground-truth distributions.

$$
L(\hat{\mathbf{y}}, \mathbf{y})=-\sum_{i=1}^{N} \sum_{j=1}^{C} y_{i}^{j} \log \left(\hat{y}_{i}^{j}\right)
$$

where $\mathbf{y}$ is the ground-truth labels, $N$ is the number of samples in the dataset, and $C$ is the number of classes. $\hat{y}_{i}^{j}$ is the probability for sample $i$ classified as class $j . y_{i}^{j}=1$ when the ground-truth class label of sample $i$ is $j$, otherwise $y_{i}^{j}=0$.

\section{COST-SENSITIVE DEEP ACTIVE LEARNING}

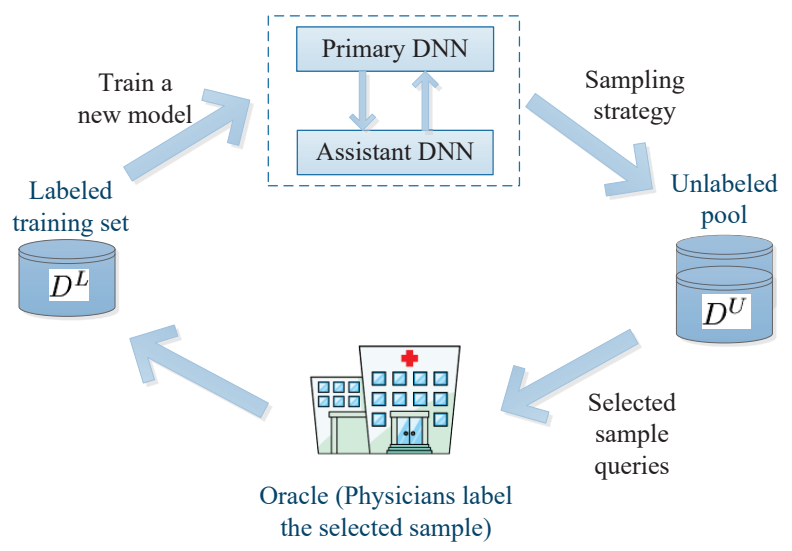

Figure 5: The structure of cost-sensitive deep active learning.

The structure of the proposed cost-sensitive deep active learning is shown in Fig. 5. A user is given a labeled dataset $D^{L}=\left\{\left(\mathbf{x}_{1}, y_{1}\right),\left(\mathbf{x}_{2}, y_{2}\right), \ldots,\left(\mathbf{x}_{n}, y_{n}\right)\right\}$ and a much large unlabeled dataset $D^{U}=\left\{\left(\mathbf{x}_{1}^{U},-\right),\left(\mathbf{x}_{2}^{U},-\right), \ldots,\left(\mathbf{x}_{n}^{U},-\right)\right\}$. In each learning iteration, a double-DNN evaluates the instances in the unlabeled data pool $D^{U}$ and selects a batch of most informative samples based on a sampling strategy. The Oracle (physician or expert) labels the selected instance and adds the labeled samples to $D^{L}$ to retrain the double-DNN. The iterations stop according to the query budget, or when the classification error meets the stopping criteria. Note that the double-DNN in this framework can employ any type deep neural network. We utilize 1D CNN, LSTM, and GRU in this study to compare their results.

In the following, we introduce the proposed double-DNN in detail and its corresponding training process.

\subsection{The Double-DNN Architecture}

The major challenge of active learning is to select the most informative instances for the current model. First, we need to pick the instances that current classifier is most uncertain about, to obtain their labels and improve the classifier. Second, when we select instances, we need to account for their different misclassification costs, which are used to be assumed identical but in fact are quite different, particularly in medical data classification. Third, we also need to consider the diversity of selected samples to avoid redundancy. To address these issues, we introduce a novel active learning architecture, called double-DNN. Specifically, the primary DNN is used as EEG classifier that generates prediction confidence, while the assistant DNN is used to predict the misclassification cost. Then, we integrate the uncertainty, misclassification cost, and diversity to construct a utility function for selecting the most informative instances in $D^{U}$, for querying the label actively.

4.1.1 Uncertainty. Uncertainty sampling [28] is one of the most popular sample selection strategies for active learning [37]. It measures the classifier's uncertainty on each instance in the unlabeled set, $D^{U}$, and then returns the most uncertain one.

In the primary DNN, We employ the least confidence to estimate the uncertainty of instances. Particularly, $\hat{y}\left(\mathbf{x}_{i}\right)$ in Eq. (16) is the posterior probability with respect to each class label. Then, the most uncertain instance is the one closest to the classification threshold $\theta$. Hence, the uncertainty can be calculated as

$$
u_{1}\left(\mathbf{x}_{i}\right)=\frac{\left\|\hat{y}\left(\mathbf{x}_{i}\right)-\theta\right\|_{2}}{\|\theta\|_{2}} .
$$

4.1.2 Misclassification Cost. Cost-sensitive learning is a type of learning in data mining that considers the misclassification costs, the goal of which is to minimize the total cost. In this paper, we extend the basic cost-sensitive learning and apply it to medical data analysis. For seizure detection, misclassifying a seizure onset sample as a normal class is more serious than misclassifying a normal sample as a seizure onset class. We define that the cost of the former should be lower than the cost of the latter since we tend to choose the instances with lowest costs. We define the cost matrix in Table 1, where "class a" refers to the seizure onset class and "class n" refers to the normal class, and $c_{1}<c_{2}<c_{3}<c_{4}$. 
Table 1: Misclassification cost matrix

\begin{tabular}{l|l}
\hline Cost & Defination \\
\hline Cost $[\mathrm{a}, \mathrm{n}]=c_{1}$ & misclassify "class a" as "class n" \\
$\operatorname{Cost}[\mathrm{n}, \mathrm{a}]=c_{2}$ & misclassify "class n" as "class a" \\
$\operatorname{Cost}[\mathrm{a}, \mathrm{a}]=c_{3}$ & correctly classify "class a" \\
$\operatorname{Cost}[\mathrm{n}, \mathrm{n}]=c_{4}$ & correctly classify "class n" \\
\hline
\end{tabular}

However, we have little knowledge about misclassification cost for the instances in set $D^{U}$. To predict the cost for these instances, we train an assistant DNN based on the output of the primary DNN. We relabel the instances in set $D^{L}$ with their misclassification cost to build a new set $D_{M_{2}}^{L}$. Then, we train the assistant DNN on $D_{M_{2}}^{L}$ to predict the misclassification cost $u_{2}\left(x_{i}\right)$ for each instance in $D^{U}$.

4.1.3 Diversity Measure. The uncertainty and misclassification cost may introduce redundancy and lead to extra labeling cost. Therefore, we also consider the diversity measure, which can be used to reduce overlap within the labeled training samples [11]. We use Euclidean distance to measure the distance between the current sample with the samples in the labeled data pool. A larger distance means more diversity among samples. Therefore, samples are chosen to maximize the overall distance between the current instance with all the previously selected instances:

$$
\sum_{\mathbf{x}_{j} \in S} d\left(\mathbf{x}_{i}, \mathbf{x}_{j}\right)=\sum_{\mathbf{x}_{j} \in S} \sqrt{\sum_{q=1}^{T}\left(x_{i q}-x_{j q}\right)^{2}},
$$

where $\mathbf{x}_{i}$ is the instance with a dimension of $T$ in the unlabeled data pool $D^{U}, \mathbf{x}_{j}$ is the instance in set $S$, which is the set of labeled batch samples in the previous AL iteration. $d(\cdot)$ is the Euclidean distance.

Besides, to normalize the distance, we divide the overall distance by the summation of the possible largest distance. Thus, the utility function $u_{3}$ is calculated as:

$$
u_{3}\left(\mathbf{x}_{i}\right)=\frac{\sum_{\mathbf{x}_{j} \in S} d\left(\mathbf{x}_{i}, \mathbf{x}_{j}\right)}{|S| * \sqrt{\sum_{q=1}^{T}\left(x_{M A X}-\left(-x_{M A X}\right)\right)^{2}}},
$$

where $|S|$ is the number of samples in set $S, x_{M A X}$ is the maximum value of ECG signals, i.e. the cutoff amplitude of EEG signals.

4.1.4 Utility Function. Finally, we explore a weighted linear combination of above three measurements to select samples for labeling. We prefer to choose the instances with higher uncertainty, lower misclassification cost, and higher diversity measures. Thus, the weighted utility function is defined as:

$$
U_{M}\left(\mathbf{x}_{i}\right)=\alpha \cdot u_{1}\left(\mathbf{x}_{i}\right)-\beta \cdot u_{2}\left(\mathbf{x}_{i}\right)+\gamma \cdot u_{3}\left(\mathbf{x}_{i}\right),
$$

where $\alpha, \beta$, and $\gamma$ are the weight parameters adjusting the influence of each measurement.
We calculate the utility score for instances in $D^{U}$ and pick the instances with highest scores to be labeled. We describe the detailed instance selection process in the following section.

\subsection{Training Process}

The details of the proposed active learning algorithm are described in Algorithm 1. We first train the primary DNN model $M_{1}$ using the labeled samples in the initial dataset $D^{L}$ with early stopping to prevent overfitting. Then, the trained $M_{1}$ predicts the label for each sample in $D^{L}$. With the predicted labels and the ground truth labels, we obtain the misclassification cost for each instance in $D^{L}$ by looking up Table 1, and accordingly build a new dataset $D_{M_{2}}^{L}$. Afterward, we train the assistant DNN $M_{2}$ with the labeled samples from dataset $D_{M_{2}}^{L}$ to learn the misclassification cost. Subsequently, for each sample in the unlabeled data pool $D^{U}$, we calculate the uncertainty score by Eq. (18) and the diversity measurement by Eq. (20), and use $M_{2}$ to predict the sample's misclassification cost. Thus, the utility for each sample in $D^{U}$ can be calculated according to Eq. (21), and the scheme is able to select the most informative instances to be labeled by Oracle. By updating $D^{L}$, we repeat the process to retrain $M_{1}$ and $M_{2}$ until the stopping criterion is met.

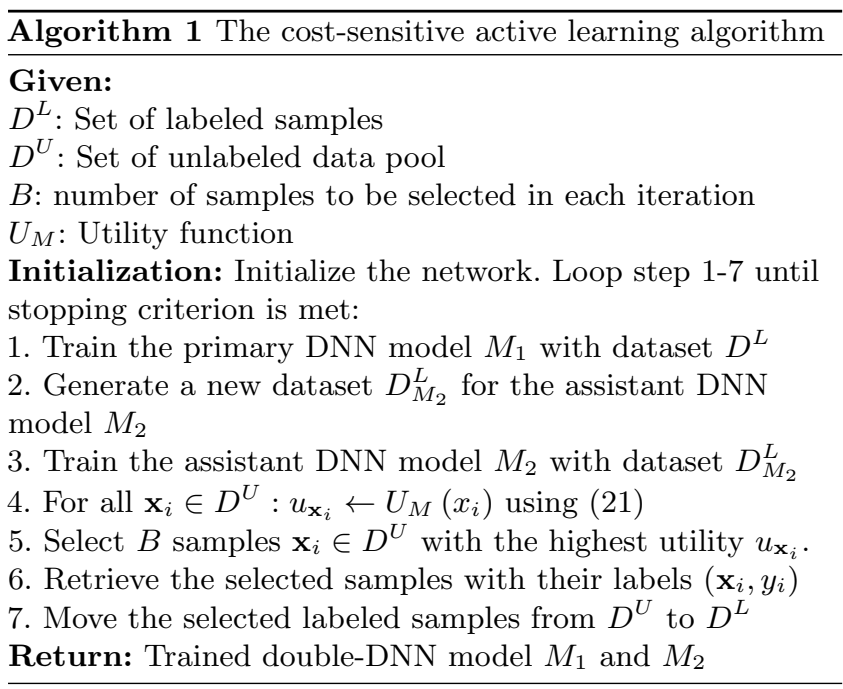

During this retraining process, the $M_{1}$ queries labels of new data from the Oracle to improve $M_{2}$ 's prediction performance. Meanwhile, more accurately predicted misclassification cost can help select the more important samples for the primary DNN to achieve a better classification results. Therefore, these two DNNs collaborate with each other to enable the system to classify the EEG signal accurately with fewer labeled samples.

\section{EXPERIMENT RESULTS}

To evaluate the proposed scheme, we employ three fundamental neural networks, i.e., 1D CNN, LSTM and GRU as 
introduced in Section 3. The details of the dataset and networks settings are as follows.

\subsection{Setup}

5.1.1 Datasets. We evaluate our double-DNN system on EEG data from Department of Epileptology, University of Bonn [5]. The entire dataset consists of five subsets, A-E, each containing 100 single channel EEG signals of 23.6 seconds duration. All EEG signals were recorded with the same 128-channel amplifier system, using an average common reference, with $12 \mathrm{~A} / \mathrm{D}$ conversion bit rate of 12 and the sampling rate of $173.61 \mathrm{~Hz}$. In our experiments, we select C, $\mathrm{D}$, and $\mathrm{E}$, three subsets as target data, which are collected from 5 epileptic patients with implanted electrodes inside their cranial. Specifically, subset $\mathrm{C}$ and D contain seizure free EEG signals, where we set the label to " $n$ ", and subset E only contains seizure onset EEG signals, where we set the label to " $a$ ".

5.1.2 Data Preprocessing. We preprocess the ECG signal to fit the data for deep learning. First, we de-noise the EEG data by applying Discrete Wavelet Transform (DWT). Specifically, to achieve a good performance, each record is processed by 5 levels discrete wavelet with Daubechies in order $4(\mathrm{db} 4)$ [3, 29]. Then we segment each 23.6 s record into 2 s length time sequences. The total 3300 segments are shuffled and divided into a training dataset and a testing dataset with $4 / 5$ and $1 / 5$ of the total segments, respectively. All the EEG segments are also normalized before feeding into the neural networks.

5.1.3 Neural Networks. We employ 1D CNN, LSTM and GRU in the proposed double-DNN, respectively, where the primary DNN and the assistant DNN always use the same type of DNN. The Adam Optimizer [24] is used with learning rate 0.001 . During the training process, the batch size is 20 , and the epoch is 10 . The misclassification costs are set as follows: $c_{1}=0.1$ for the predicted label is " $n$ " while the true label is " $a$ ", $c_{2}=0.2$ for the predicted label is " $a$ " while the true label is " $n$ ", $c_{3}=0.4$ for the predicted label is " $a$ " while the true label is " $a$ ", and $c_{4}=0.5$ for the predicted label is " $n$ " while the true label is " $n$ ". The weights in the utility function are chosen according to experimental trials, where the parameters change from 0.1 to 0.8 and add up to 1 . There are slight differences among different trials, and we set the parameters to $\alpha=0.4, \beta=0.4$, and $\gamma=0.2$. We label 100 segments in the labeled data pool as the initial training dataset, where label " $n$ " for segments from subset C and D, and label " $a$ " for segments from subset E. In each iteration, the query strategy select 20 instances from unlabeled data pool to query the oracle. The iterations stop until there are no samples in the unlabeled data pool.

For the primary and secondary neural networks in the double-CNN system, we used a one-dimensional CNN with $1 \times 10$ filters, a stride of 1 and mapcount of 10 . The onedimensional CNN is followed by rectifier activations and a global max pooling layer. The final fully connected layer uses sigmoid activations.

For the primary and secondary neural networks in the double-LSTM system, we use one recurrent neural layer with 256 LSTM nodes and one fully connected layer with sigmoid activations. The settings of double-GRU system are similar to those in the double-LSTM system.

\subsection{Results}

Table 2: Seizure detection performance of different machine learning schemes

\begin{tabular}{|l|l|l|l|}
\hline Classifier & Precision & Recall & Accuracy \\
\hline Decision Tree & $86.00 \%$ & $85.45 \%$ & $90.52 \%$ \\
Logistic Classification & $84.88 \%$ & $38.27 \%$ & $77.15 \%$ \\
Gaussian SVM & $95.42 \%$ & $87.09 \%$ & $94.30 \%$ \\
KNN & $99.47 \%$ & $68.45 \%$ & $89.36 \%$ \\
Ensemble Boosted Trees & $96.99 \%$ & $85.09 \%$ & $94.15 \%$ \\
Ensemble Bagged Trees & $95.21 \%$ & $92.27 \%$ & $95.88 \%$ \\
1D CNN & $99.54 \%$ & $92.37 \%$ & $97.27 \%$ \\
LSTM & $97.75 \%$ & $92.55 \%$ & $96.82 \%$ \\
GRU & $99.08 \%$ & $91.53 \%$ & $96.67 \%$ \\
\hline
\end{tabular}

In this section, we first compare the epileptic seizure detection performance of deep learning and classical machine learning including decision tree, logistic classification, Gaussian support vector machine, k-nearest neighbor, ensemble boosted trees, and ensemble bagged tree. Specifically, for deep learning methods, we consider $1 \mathrm{D}$ CNN, RNN with LSTM and RNN with GRU. We use 5-fold cross-validation to evaluate the performance of each method. Then we take precision, recall and accuracy as metrics, and the results are shown in Table 2. Precision is the ratio that the number of correctly classified seizure onset over the total number of classified seizure onset. Recall measures the ratio of correctly predicted seizures to the number of actual seizures. Overall accuracy is the ratio that the number of correctly classified samples over the number of all testing samples. The highest overall accuracy among the classical machine learning schemes is $95.88 \%$ from Bagged Tree, while the three DNNs are $97.27 \%, 96.82 \%$, and $96.67 \%$, respectively. The highest precision is $99.47 \%$ from $\mathrm{KNN}$, while the deep learning methods achieve $99.45 \%, 97.75 \%$, and $99.08 \%$, respectively. Nevertheless, compared to classical machine learning methods, the deep learning methods have better recall with $92.37 \%$, $92.55 \%$ and $91.53 \%$, receptively. As discussed in Section 1, the physicians pay more attention to the accuracy of the seizure onset samples. Thereby, the higher recall is preferred in the classification system. So, the deep neural networks achieve better performance in seizure detection. Moreover, the DNNs can save the manual feature selection and extraction process, and thus reduce the human efforts.

Then we evaluate the efficacy and efficiency of our proposed double-DNN system with three fundamental networks, 1D CNN, RNN with LSTM and RNN with GRU. We compare our active learning methods with uncertainty sampling 

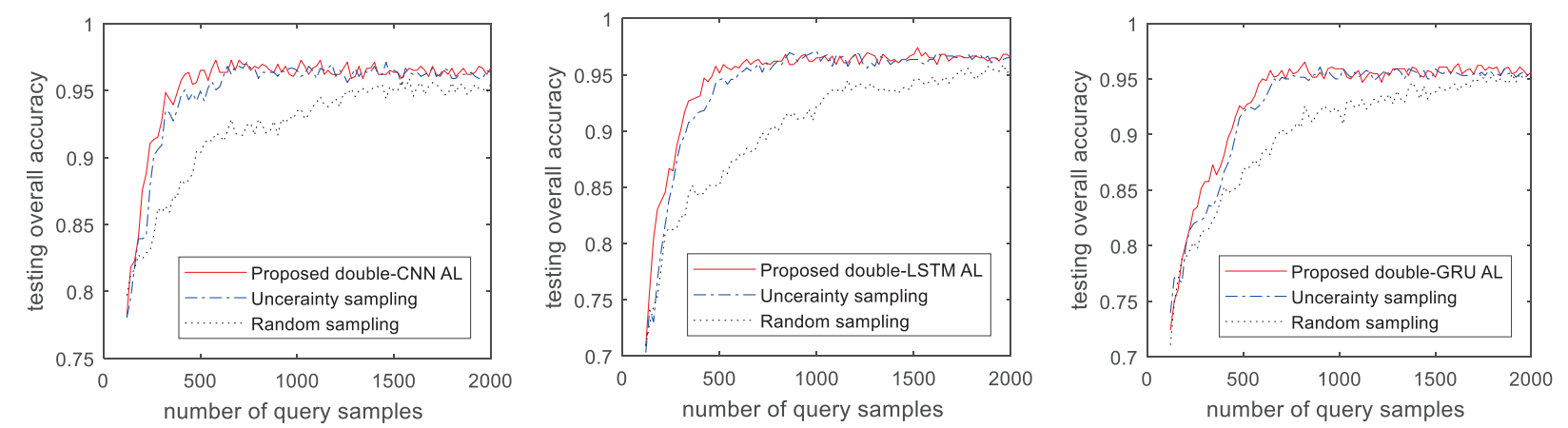

(a) The overall detection accuracy with $1 \mathrm{D}(\mathrm{b})$
CNN.

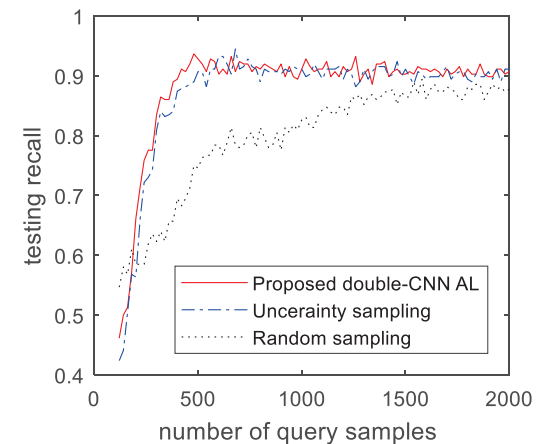

(d) The recall of seizure onset with 1D CNN.
M.

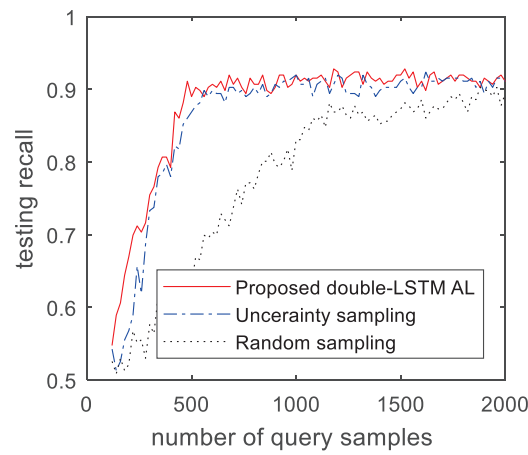

(e) The recall of seizure onset with LSTM. (c) The overall detection accuracy with GRU.

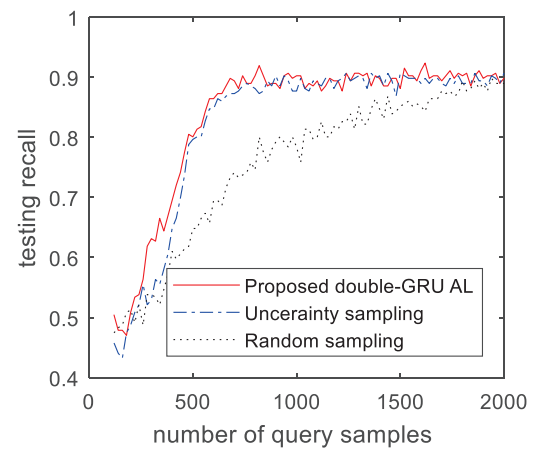

(f) The recall of seizure onset with GRU.

\section{Figure 6: The performance evaluation of double-DNNs}

methods and random sampling methods. First, we evaluate the overall accuracy in Fig. 6(a), Fig. 6(b) and Fig. 6(c). Taking Fig. 6(a) as an example, with the number of query samples increasing, the detection accuracy improves. The proposed strategy grows dramatically at the beginning and converges at 400 query samples with the accuracy around $96.5 \%$. The uncertainty sampling scheme converges at 600 query samples, and the random sampling is not converged after 2000 query samples. Thus, the proposed scheme can reduce the amount of labeled samples by 33\% and $80 \%$ compared with uncertainty sampling and random sampling, respectively. In addition, when the labeling query budget is limited (less than 600), the proposed scheme can achieve the highest accuracy. The same conclusion can be made on RNN with LSTM in Fig. 6(b) and RNN with GRU in Fig. 6(c).

As discussed in Section 4, in practice, we focus on mining seizure onset class. Thus, we evaluate the recall, which measures the detection accuracy of seizure onset EEG signals, shown in Fig. 6(d), Fig. 6(e) and Fig. 6(f). Still, we take $1 \mathrm{D} \mathrm{CNN}$ as an example, the proposed active learning outperforms the random sampling and uncertainty sampling. The proposed double-CNN converges at 400 query samples with $92 \%$ recall, while the uncertainty needs 600 query samples. The misclassification cost urges the network to select samples that tend to have less cost in the unlabeled data pool, which means the scheme tends to choose seizure onset instance that tends to be classified as normal EEG activities. Hence, the double-CNN scheme obtains a higher true positive rate for seizure class with the same number of query samples. The same conclusion can be made on RNN with LSTM in Fig. 6(e) and RNN with GRU in Fig. 6(f).

We also compare the performance of these three DNNs directly in Fig. 7. In this dataset, the double-CNN achieves the best performance with the fastest converge rate, which has better detection accuracy with insufficient labeling samples, especially under 400 samples.

To sum up, our proposed double-DNN achieves the highest seizure detection accuracy with $97.28 \%$, and the proposed scheme can reduce the amount of labeled samples by $33 \%$ and $80 \%$ compared with uncertainty sampling and random sampling, respectively. We also compare the performance for three DNNs and discover that the double-CNN achieves the best performance.

\section{CONCLUSIONS AND DISCUSSIONS}

In this paper, we propose a deep active learning framework for epileptic seizure detection using EEG signals. Since misclassification for normal and abnormal signals have different costs which are however unknown, we propose a generic double-DNN sample selection strategy that can not only 


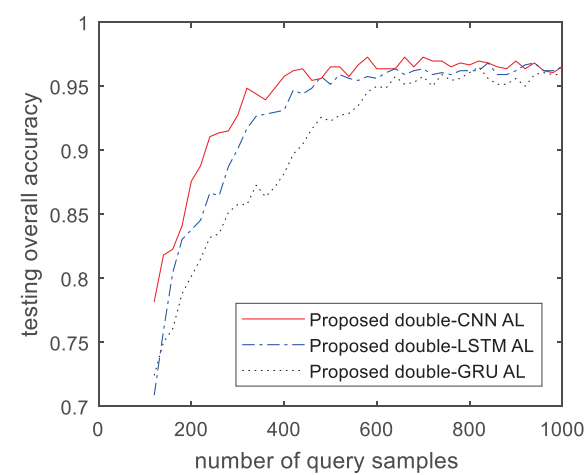

Figure 7: The performance of double-1D CNN, double-LSTM, and double-GRU on seizure onset detection.

classify EEG signals but also predict their misclassification costs by taking into account different costs for different type$\mathrm{s}$ of misclassifications. Experiment results demonstrate that our proposed scheme achieves the better performance than the uncertainty sampling and random sampling strategies. Particularly, our proposed scheme can have a more accurate classifier compared with the baselines and can converge with a much similar amount of samples. Meanwhile, the proposed scheme also achieve higher accuracy compared with traditional machine learning schemes.

Besides, the proposed scheme can be generalized to other problems. This paper focuses on epilepsy seizure detection, where the seizure onset activity is more important than the normal activity, and thus has the cost-sensitive problem. In other domains, like cardiac arrhythmia classification, the same situation also exists. For instance, the premature ventricular contraction (PVC) heartbeats, which need immediate medical care, is much more serious than atrial premature (AP) heartbeats, which are quite normal and do not need immediate attention. Thus, we can implement the costsensitive active learning scheme to efficiently train a heartbeat classifier that focuses more on the severe arrhythmia types.

In addition, the selection of deep neural networks in the proposed scheme may vary in different problems. For example, 1D CNN obtains the best performance on EEG datasets as shown in this study, but it may not be the best on ECG datasets. Therefore, we provide and evaluate three types of DNNs in this paper as examples, which hopefully provide insights when readers choose their own DNNs in other problems.

\section{ACKNOWLEDGMENTS}

This work was partially supported by the U.S. National Science Foundation under grants CNS-1602172 and CNS1566479 .

\section{REFERENCES}

[1] U Rajendra Acharya, Filippo Molinari, S Vinitha Sree, Subhagata Chattopadhyay, Kwan-Hoong Ng, and Jasjit S Suri. 2012. Automat ed diagnosis of epileptic EEG using entropies. Biomedical Signal Processing and Control 7, 4 (2012), 401-408.

[2] U Rajendra Acharya, S Vinitha Sree, Subhagata Chattopadhyay, Wenwei Yu, and Peng Chuan Alvin Ang. 2011. Application of recurrence quantification analysis for the automated identification of epileptic EEG signals. International journal of neural systems 21, 03 (2011), 199-211.

[3] Hojjat Adeli, Ziqin Zhou, and Nahid Dadmehr. 2003. Analysis of EEG records in an epileptic patient using wavelet transform. Journal of neuroscience methods 123, 1 (2003), 69-87.

[4] Xiu An, Deping Kuang, Xiaojiao Guo, Yilu Zhao, and Lianghua He. 2014. A deep learning method for classification of EEG data based on motor imagery. In International Conference on Intelligent Computing. Springer, 203-210.

[5] Ralph G Andrzejak, Klaus Lehnertz, Florian Mormann, Christoph Rieke, Peter David, and Christian E Elger. 2001. Indications of nonlinear deterministic and finite-dimensional structures in time series of brain electrical activity: Dependence on recording region and brain state. Physical Review E 64, 6 (2001), 061907.

[6] Anthony C Atkinson and RA Bailey. 2001. One hundred years of the design of experiments on and off the pages of Biometrika. Biometrika 88, 1 (2001), 53-97.

[7] Guha Balakrishnan and Zeeshan Syed. 2010. Scalable personalized medicine with active learning: Detecting seizures with minimum labeled data. In Proceedings of the 1st ACM International Health Informatics Symposium. ACM, 83-90.

[8] Guha Balakrishnan and Zeeshan Syed. 2012. Scalable personalization of long-term physiological monitoring: Active learning methodologies for epileptic seizure onset detection. In Artificial Intelligence and Statistics. 73-81.

[9] Pouya Bashivan, Irina Rish, Mohammed Yeasin, and Noel Codella. 2015. Learning representations from EEG with deep recurrentconvolutional neural networks. arXiv preprint arXiv:1511.06448 (2015).

[10] ANN BENSCHOTER. [n. d.]. What is Epilepsy. https://www.cureepilepsy.org/about-epilepsy/what-is-epilepsy. Accessed: 2018-05-18.

[11] Klaus Brinker. 2003. Incorporating diversity in active learning with support vector machines. In Proceedings of the 20th international conference on machine learning (ICML-03). 59-66.

[12] Xuhui Chen, Jinlong Ji, Kenneth Loparo, and Pan Li. 2017. Realtime personalized cardiac arrhythmia detection and diagnosis: A cloud computing architecture. In Biomedical $\&$ Health Informatics (BHI), 2017 IEEE EMBS International Conference on. IEEE, 201-204.

[13] Kyunghyun Cho, Bart Van Merriënboer, Caglar Gulcehre, Dzmitry Bahdanau, Fethi Bougares, Holger Schwenk, and Yoshua Bengio. 2014. Learning phrase representations using RNN encoderdecoder for statistical machine translation. arXiv preprint arXiv:1406.1078 (2014).

[14] Junyoung Chung, Caglar Gulcehre, KyungHyun Cho, and Yoshua Bengio. 2014. Empirical evaluation of gated recurrent neural networks on sequence modeling. arXiv preprint arXiv:1412.3555 (2014).

[15] Ronan Collobert, Jason Weston, Léon Bottou, Michael Karlen, Koray Kavukcuoglu, and Pavel Kuksa. 2011. Natural language processing (almost) from scratch. Journal of Machine Learning Research 12, Aug (2011), 2493-2537.

[16] PR Davidson, RD Jones, and MTR Peiris. 2006. Detecting behavioral microsleeps using EEG and LSTM recurrent neural networks. In Engineering in Medicine and Biology Society, 2005. IEEE-EMBS 2005. 27th Annual International Conference of the. IEEE, 5754-5757.

[17] Jeffrey L Elman. 1990. Finding structure in time. Cognitive science 14, 2 (1990), 179-211.

[18] Yarin Gal, Riashat Islam, and Zoubin Ghahramani. 2017. Deep Bayesian Active Learning with Image Data. arXiv preprint arXiv:1703.02910 (2017).

[19] Sepp Hochreiter and Jürgen Schmidhuber. 1997. Long short-term memory. Neural computation 9, 8 (1997), 1735-1780.

[20] Baotian $\mathrm{Hu}$, Zhengdong Lu, Hang Li, and Qingcai Chen. 2014. Convolutional neural network architectures for matching natural 
language sentences. In Advances in neural information processing systems. 2042-2050.

[21] Jinlong Ji, Xuhui Chen, Changqing Luo, and Pan Li. 2018. A deep multi-task learning approach for ECG data analysis. In Biomedical \& Health Informatics (BHI), 2018 IEEE EMBS In ternational Conference on. IEEE, 124-127.

22] Jinlong Ji, Changqing Luo, Xuhui Chen, Lixing Yu, and Pan Li. 2018. Cross-Domain Sentiment Classification via a BifurcatedLSTM. In Pacific-Asia Conference on Knowledge Discovery and Data Mining. Springer, 681-693.

23] Xiaowei Jia, Kang Li, Xiaoyi Li, and Aidong Zhang. 2014. A novel semi-supervised deep learning framework for affective state recognition on eeg signals. In Bioinformatics and Bioengineering (BIBE), 2014 IEEE International Conference on. IEEE, 30-37.

[24] Diederik P Kingma and Jimmy Ba. 2014. Adam: A method for stochastic optimization. arXiv preprint arXiv:1412.6980 (2014).

25] Vernon Lawhern, David Slayback, Dongrui Wu, and Brent J Lance. 2015. Efficient labeling of EEG signal artifacts using active learning. In Systems, Man, and Cybernetics (SMC), 2015 IEEE International Conference on. IEEE, 3217-3222.

[26] Yann LeCun, Yoshua Bengio, and Geoffrey Hinton. 2015. Deep learning. nature 521, 7553 (2015), 436.

[27] Yann LeCun, Léon Bottou, Yoshua Bengio, and Patrick Haffner 1998. Gradient-based learning applied to document recognition. Proc. IEEE 86, 11 (1998), 2278-2324.

[28] David D Lewis and Jason Catlett. 1994. Heterogeneous uncertainty sampling for supervised learning. In Proceedings of the eleventh international conference on machine learning. 148156.

[29] Mingyang Li, Wanzhong Chen, and Tao Zhang. 2017. Classification of epilepsy EEG signals using DWT-based envelope analysis and neural network ensemble. Biomedical Signal Processing and Control 31 (2017), 357-365.

[30] Peng Liu, Hui Zhang, and Kie B Eom. 2017. Active deep learning for classification of hyperspectral images. IEEE Journal of Selected Topics in Applied Earth Observations and Remote Sensing 10, 2 (2017), 712-724.

[31] LR Medsker and LC Jain. 2001. Recurrent neural networks. De sign and Applications 5 (2001).

[32] Andrew W Moore, Jeff G Schneider, Justin A Boyan, and Mary S Lee. 2000. Q2: Memory-based active learning for optimizing noisy continuous functions. In Robotics and Automation, 2000. Proceedings. ICRA'00. IEEE International Conference on, Vol. 4 IEEE, 4095-4102.

[33] Umut Orhan, Mahmut Hekim, and Mahmut Ozer. 2011. EEG signals classification using the K-means clustering and a multilayer perceptron neural network model. Expert Systems with Applications 38, 10 (2011), 13475-13481.

[34] Kemal Polat and Salih Güneş. 2007. Classification of epileptiform EEG using a hybrid system based on decision tree classifier and fast Fourier transform. Appl. Math. Comput. 187, 2 (2007), 10171026.

[35] Robin Tibor Schirrmeister, Jost Tobias Springenberg, Lukas Dominique Josef Fiederer, Martin Glasstetter, Katharina Eggensperger, Michael Tangermann, Frank Hutter, Wolfram Burgard, and Tonio Ball. 2017. Deep learning with convolutional neural networks for EEG decoding and visualization. Human brain mapping 38, 11 (2017), 5391-5420.

[36] Burr Settles. 2010. Active learning literature survey. University of Wisconsin, Madison 52, 55-66 (2010), 11.

[37] Burr Settles. 2012. Active learning. Synthesis Lectures on Artificial Intelligence and Machine Learning 6, 1 (2012), 1-114.

[38] V Srinivasan, C Eswaran, Sriraam, and N. 2005. Artificial neural network based epileptic detection using time-domain and frequency-domain features. Journal of Medical Systems 29, 6 (2005), 647-660.

[39] Vairavan Srinivasan, Chikkannan Eswaran, and Natarajan Sriraam. 2007. Approximate entropy-based epileptic EEG detection using artificial neural networks. IEEE Transactions on informa tion Technology in Biomedicine 11, 3 (2007), 288-295.

[40] Sebastian Stober, Daniel J Cameron, and Jessica A Grahn. 2014 Using Convolutional Neural Networks to Recognize Rhythm Stimuli from Electroencephalography Recordings. In Advances in neural information processing systems. 1449-1457.

[41] Sebastian Stober, Avital Sternin, Adrian M Owen, and Jessica A Grahn. 2015. Deep feature learning for EEG recordings. arXiv preprint arXiv:1511.04306 (2015).
[42] Keze Wang, Dongyu Zhang, Ya Li, Ruimao Zhang, and Liang Lin. 2016. Cost-effective active learning for deep image classification. IEEE Transactions on Circuits and Systems for Video Technology (2016).

[43] Dongrui Wu, Brent Lance, and Vernon Lawhern. 2014. Transfer learning and active transfer learning for reducing calibration data in single-trial classification of visually-evoked potentials. In Systems, Man and Cybernetics (SMC), 2014 IEEE International Conference on. IEEE, 2801-2807.

[44] Dongrui Wu, Vernon J Lawhern, Stephen Gordon, Brent J Lance, and Chin-Teng Lin. 2016. Offline EEG-based driver drowsiness estimation using enhanced batch-mode active learning (EBMAL) for regression. In Systems, Man, and Cybernetics (SMC), 2016 IEEE International Conference on. IEEE, 000730-000736.

[45] Dongrui Wu, Vernon J Lawhern, W David Hairston, and Brent J Lance. 2016. Switching EEG headsets made easy: Reducing offline calibration effort using active weighted adaptation regularization. IEEE Transactions on Neural Systems and Rehabilitation Engineering 24, 11 (2016), 1125-1137.

[46] Wei-Long Zheng, Jia-Yi Zhu, Yong Peng, and Bao-Liang Lu. 2014. EEG-based emotion classification using deep belief networks. In Multimedia and Expo (ICME), 2014 IEEE International Conference on. IEEE, 1-6. 